\title{
History-dependent growth incidence: a characterization and an application to the economic crisis in Italy
}

\author{
By Flaviana Palmisano ${ }^{a}$ and Dirk Van de gaer $^{b}$
}

\author{
aniversity of Luxembourg, Maison des Sciences Humaines, 11 Porte des Sciences L-4366 \\ Esch-sur-Alzette, Luxembourg and LUMSA, Rome; e-mail: flaviana.palmisano@gmail.com \\ ${ }^{b}$ Sherppa, Ghent University; IAE, CSIC, Barcelona; and CORE, Université Catholique de Louvain; \\ e-mail: Dirk.Vandegaer@ugent.be
}

\begin{abstract}
We propose a characterization of an aggregate measure of growth that takes into account the initial economic conditions of individuals. Our measure is a weighted average of individuals' income growth with weights that are decreasing with the rank of the individual in the initial income distribution. We apply our theoretical framework to evaluate the growth processes experienced by the Italian population. Even when we correct for the differences in mean income growth, and focus on the distribution of growth only, the 2008-2010 growth process was the worst of the last decade.
\end{abstract}

JEL classifications: D31, D63, I32

\section{Introduction}

Eventful days such as the different phases of the recent economic crisis rapidly follow each other. These events motivate a renewed and increasing interest in the measurement of growth and its distributional implications. We focus on the question of how the financial crisis, compared to the growth processes preceding it, affected the distribution of income growth when a higher weight is given to the income growth of the initially poor, and after adjusting for differences in mean growth.

We adopt a history-dependent perspective, which evaluates a growth process on the basis of individuals' growth experiences and their position in the initial distribution of income. Such approaches are becoming increasingly popular (Grimm, 2007; Van Kerm, 2009; Bourguignon, 2011; Jenkins and Van Kerm, 2011; Palmisano and Peragine, 2015). These approaches mainly employ the 'Non-anonymous Growth Incidence Curve' (na-GIC; see Bourguignon, 2011) also called the 'Mobility Profile' (Van Kerm, 2009), which plots the growth in mean income of those individuals belonging to the same quantile in the initial distribution of income as a function of their quantile in this initial distribution. 

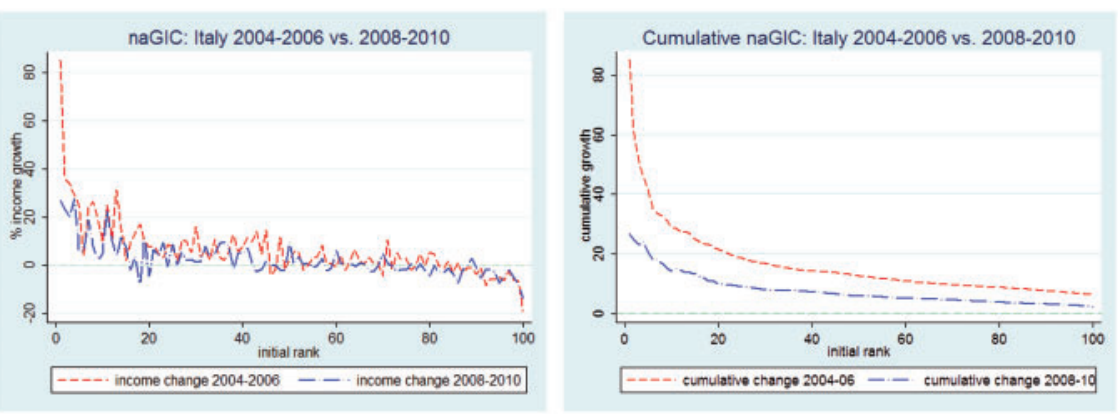

Fig. 1. Panel (a) na-GICs and (b) cumulative na-GICs for Italy

Notes: Computations of the (cumulative) na-GICs are based on the mean growth obtained by each percentile in the initial income distribution. Authors' calculations, based on the Italian 'Survey on Household Income and Wealth'.

The literature cited above provides formal derivations of dominance conditions that can be used to obtain incomplete rankings of growth processes. A first dominance criterion is that one growth process is better than another if its na-GIC lies above the other's na-GIC. In practice, such dominance is rather exceptional, as illustrated in panel (a) of Fig. 1, which plots the Italian na-GICs for the period before (2004-06, dashed line) and after (2008-10, dashed-dot line) the financial crisis.

Both na-GICs are positive up to the 50th percentile, are around zero up to the 85 th percentile, and become negative for the initially richest percentiles. Hence, in both periods the incomes of the initially poorest grew more than those of the initially rich. However, we encounter a major difficulty in the comparison of these two growth processes. No dominance can be established since the two curves intersect very often.

A second, more powerful dominance condition can be obtained when one is willing to attach a greater weight to the growth rates of the initially poor. In that case, one growth process is better than another if its cumulative na-GIC lies above the other's cumulative naGIC. Figure 1, panel (b) plots the cumulative na-GICs for the two growth processes and, in this case, we obtain a clear ranking: the growth process from 2004-06 was unambiguously better than the growth process from 2008-10.

There are several issues worth pointing out in the procedure outlined so far, however. First, dominance between cumulative na-GICs cannot always be established. In the analyses performed by Bourguignon (2011) and Jenkins and Van Kerm (2011), for instance, dominance between cumulative na-GICs is the exception rather than the rule.

Second, the computation of na-GICs requires either that the data are arbitrarily partitioned into quantiles, or a non-trivial estimation of the na-GIC (on the latter, see Jenkins and Van Kerm, 2011). The choice of which quantile to use is arbitrary, and dominance is more likely to be obtained with a coarse rather than a fine partitioning of the data. Moreover, as individual data are necessary as the input in the entire procedure, we believe it is preferable to compute na-GICs and cumulative na-GICs directly from the individual data. This is done in Fig. 2.

The individual na-GIC depicts each individual's growth rate as a function of his relative position in the initial income distribution. As can be seen in panel (a) of Fig. 2, these individual na-GICs intersect very often. Panel (b) shows that the cumulative individual na-GICs also intersect. 

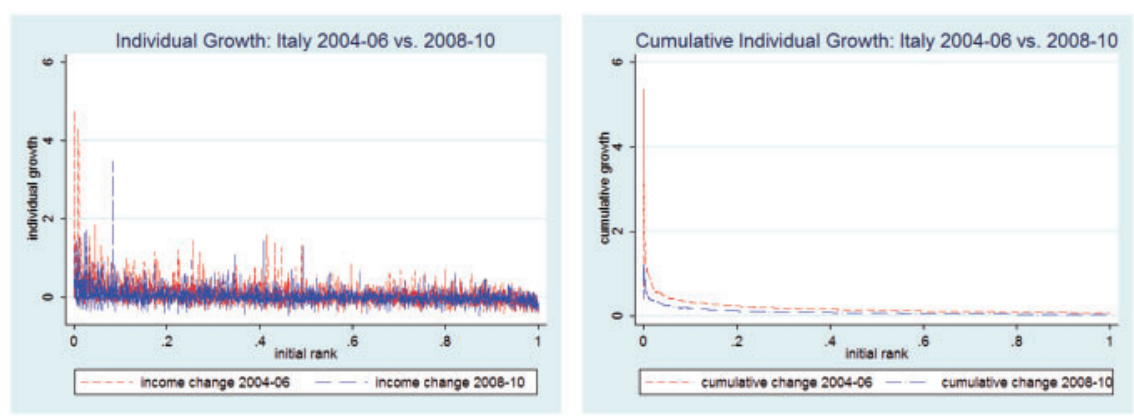

Fig. 2. Panel (a) individual na-GICs and (b) cumulative individual na-GICs for Italy

Notes: Computations of the (cumulative) individual na-GICs are based on the growth obtained by each individual as a function of his relative rank in the initial income distribution. Authors' calculations, based on the Italian 'Survey on Household Income and Wealth'.

Third, it has to be pointed out that comparing (individual) na-GICs and cumulative naGICs is heavily influenced by the mean of the individual income growth. One might be interested in the purely redistributive aspect of the growth processes, i.e., in a comparison of the growth processes after correcting for differences in the mean of individual income growth.

We propose to deal with these issues by providing an index that embodies the intuition of the na-GIC and focus exclusively on the history dependent-perspective. With the exceptions of Jenkins and Van Kerm (2011) and Genicot and Ray (2013), there does not exist a synthetic index of history-dependent growth. Our index is a weighted average of individuals' income growth with weights that are decreasing with the rank in the initial distribution of income, while Genicot and Ray's index weights an individual's growth rate based on the level of his initial income. Therefore, our index is more directly related to the naGIC. We show that, like the Jenkins and Van Kerm index, our index is additively decomposable into a progressivity index, measuring the pure redistributive effect of the growth process, and the mean of individuals' growth. Actually, it turns out that the discrete version of the Jenkins and Van Kerm index is an approximation to our index. Finally, in the empirical application, despite the frequently crossing individual na-GICs, a clear ranking of the growth processes before and after the financial crisis in Italy appears: the financial crisis disproportionately depressed the growth experiences of the initially poor.

To operationalize the framework, we first need a measure of individual income growth. We characterize two standard measures: the proportional and level change in income. Both satisfy Normalization, Monotonicity, and a Resourcist Principle. Normalization (N) and Monotonicity (M) are common properties in the literature. The Resourcist Principle (RP) is a new property; it requires that if two individuals with the same initial income see their resources (incomes) increase by the same amount, then the effect on these individuals' growth values should be the same. Standard Scale Invariance (SI) and Addition Invariance (AI), are then imposed to obtain the specific functional form of both measures of individual growth.

In the axiomatic characterization of our aggregate measure of history-dependent growth, a first ingredient is the Rank Dependent Monotonicity (RDM) axiom, which says that aggregate growth is an increasing function of individuals' growth values, ordered on the basis of individuals' initial income. A second ingredient is the History Dependent Growth Incidence (HDGI) axiom, which says that we like redistributions of individual 
growth values in favor of those having a low level of initial income, and are indifferent between growth redistributions among individuals having the same initial level of income. The latter guarantees that we do not treat the growth values of two individuals with the same initial income level differently. Next, we impose the counterparts of the structural axioms used by Bossert (1990) and Demuynck and Van de gaer (2012) on the present domain, the domain of initial income level based averaged growth, ranked on the basis of initial income. In particular, we impose Relative and Translation Invariance (RI and TI), requiring that the aggregate growth ordering of two growth processes is unaffected when, in both processes, all individuals' growth values are multiplied by the same constant or when, in both growth processes, the same constant is added to all individuals' growth values, respectively. We then impose Highest Initial Income Groups Separability (HIIGS), which requires that, for any $n \geq 2$, aggregate growth only depends on the aggregate growth of the $n-1$ group of initially richest, and on the growth of the initially poorest. Within all highest initial income groups, the trade-offs between the growth values of individuals with different initial incomes remains the same as more and more growth values of individuals with lower initial incomes are added. As such, the judgment of what happens to the growth values of any group containing the initially richest individuals (e.g., those with an initial income above a certain cut-off) does not depend on the growth values of those that were initially poorer. This makes it meaningful to study what happens to the growth values of those that had an income above the cut-off. This separability axiom is weaker than other separability axioms used in the mobility literature, such as subgroup consistency (see, e.g., Fields and Ok, 1999b; D’Agostino and Dardanoni, 2009; and Schluter and Van de gaer, 2011). Further imposing Population Invariance (PI), we obtain our aggregate index of history-dependent growth.

Two remarks are in order at this stage. First, note that, from a formal point of view, the contribution of our work to the existing literature is twofold. The first is that we provide a unifying framework for the derivation of an absolute and a relative measure of individual growth. The second is the aggregation procedure, which is similar to the one leading to the single-series Gini of Donaldson and Weymark (1980). Second, note that the history-dependent perspective is different from the pro-poor perspective, which looks at the extent to which poverty declines over time. The main instrument in this literature is the 'Growth Incidence Curve' (GIC), which plots the growth in mean income at the same percentile in the income distributions in two consecutive periods as a function of this percentile (Ravallion and Chen, 2003; Son, 2004). Contrary to the history-dependent perspective, incomes of different individuals are used to compute the growth in mean incomes, as those that are at a particular percentile in the initial income distribution are not necessarily at that same percentile in the second-period income distribution. A variety of indices for the measurement of pro-poor growth is available (see Kakwani and Pernia, 2000; Essama-Nssah, 2005; Kraay, 2006; Grosse et al., 2008; Kakwani and Son, 2008; Essama-Nssah and Lambert, 2009). However, as already pointed out in a seminal paper by Jenkins and Van Kerm (2006), this kind of analysis of income distribution trends that is based on cross-sectional data sets, ignores the reshuffling of individuals in the income distribution over time.

We end the paper with an empirical illustration of our theoretical framework that aims at comparing different consecutive two-year growth processes that took place in Italy from 1998 against the growth process 2008-2010. The focus on 2008-2010 stems from the observation that this is the period during which the first wave of the economic crisis took place. 
The paper is organized as follows. In Section 2 we introduce the general notation and present our theoretical results. Section 3 applies the framework to the recent economic crisis in Italy (2008-10). Section 4 concludes.

\section{The framework}

In this section we characterize two individual measures of growth and the aggregation of these measures into a societal index of history-dependent growth. We follow the major branch in the literature on income mobility measurement by working with a set of observations of individuals' incomes in two periods (see, e.g. Fields and Ok, 1999a). This approach has the main advantage that we use the income data in the way they are reported in panel data sets; we do not aggregate them into arbitrary quantiles and compute our index directly on the basis of the individual data. We start by defining the notation we will use throughout this paper.

Let the set of natural numbers be $\mathbb{N}, \mathbb{N}_{0}=\mathbb{N} \backslash\{0\}$ and $\mathbb{N}_{0,1}=\mathbb{N} \backslash\{0,1\}$. The set of individuals is $N=\{1, \ldots, n\} ; n \in \mathbb{N}_{0}$ is the number of individuals in society. Further, $\mathbb{R}$ is the set of real numbers, $\mathbb{R}^{+}$is the set of positive real numbers, $\mathbb{R}_{0}=\mathbb{R} \backslash\{0\}$ and $\mathbb{R}_{0}^{+}=\mathbb{R}^{+} \backslash\{0\}$. The $n$ - fold Cartesian product of a set $X$ is denoted by $X^{n}$. Individual $i$ 's initial (first period) income is $x_{i}$, and his second-period income is $w_{i}$. As the history-dependent growth perspective evaluates a growth process on the basis of individuals' growth values, we have to keep track of which individual received which income in each period, and of every individual's position in the initial distribution of income. Hence, for each $n \in \mathbb{N}_{0}$ we focus on the domain

$$
\hat{D}^{n}=\left\{\left(x_{1}, \ldots, x_{i}, \ldots, x_{n}, w_{1}, \ldots, w_{i}, \ldots, w_{n}\right) \in\left(\mathbb{R}_{0}^{+}\right)^{2 n} \text { such that } x_{1} \geq \ldots \geq x_{n}\right\} .
$$

Our aim is to characterize a sequence of aggregate growth measures $\left\{G^{n}\right\}_{n \in \mathbb{N}_{0}}$, $G^{n}: \hat{D}^{n} \rightarrow \mathbb{R}$, where each $G^{n}$ is a continuous non-constant function that measures aggregate growth in a population of size $n \in \mathbb{N}_{0}$, with special case $G^{1}$ measuring the growth value of an individual, for which the domain, $\hat{D}^{1}$, reduces to $\left(\mathbb{R}_{0}^{+}\right)^{2}$. In the first subsection, we characterize two measures of individual growth that have been used extensively in empirical works. The second subsection deals with the aggregation of these individual growth values into a societal measure of history-dependent growth. All proofs are gathered in the Appendix A.

\subsection{Individual growth}

We propose a relative and an absolute measure of individual growth. There are good reasons to use one or the other. A discussion of their pros and cons for the measurement of growth in a history-dependent context is outside the scope of this work, however. ${ }^{1}$

Three axioms will be used to characterize both a relative and an absolute measure of individual growth. The first is a standard normalization axiom, which requires that a measure of individual growth should be equal to 0 if the individual does not experience any variation in her income.

$\mathbf{N}$ (Normalization): For all $x \in \mathbb{R}_{0}^{+}: G^{1}(x, x)=0$.

The second axiom is a trivial monotonicity axiom: growth is increasing in second-period income.

1 For a detailed analysis of this issue in the context of income inequality measurement, see Kolm (1976a and 1976b) and Atkinson and Brandolini (2010). 
M (Monotonicity): For all $x, w, z \in \mathbb{R}_{0}^{+}: w>z \Rightarrow G^{1}(x, w)>G^{1}(x, z)$.

The third axiom is a resourcist principle. Increasing an individual's second-period income by a certain amount requires the same amount of monetary resources, irrespective of the level of second-period income he already has. Hence, we require that the effect of increases in second-period income on individual growth must be independent of the level of second-period income.

RP (Resourcist Principle): For all $x, w, z, \in \mathbb{R}_{0}^{+}$and $\theta>0$ :

$$
G^{1}(x, w+\theta)-G^{1}(x, w)=G^{1}(x, z+\theta)-G^{1}(x, z) .
$$

This axiom rules out considerations like decreasing contributions of second period income to individual growth, and makes clear that our measure is not a measure of welfare growth, but a measure of resource growth. It implies that the individual income growth measure is additive in second period income. This axiom will be used to cardinalize both the relative and absolute measure. Axiom $\mathrm{N}$ provides further restrictions on the cardinalization.

2.1.1 A measure of relative growth As is standard, measures of relative growth are scaleinvariant measures: they are not affected by an equiproportional change in the initial and final level of income.

SI (Scale Invariance): For all $\lambda>0$ and all $x, w \in \mathbb{R}_{0}^{+}$:

$$
G^{1}(\lambda x, \lambda w)=G^{1}(x, w) .
$$

It is easy to obtain the following Lemma.

Lemma 1 For all $x, v, w, z \in \mathbb{R}_{0}^{+}$the individual growth measure satisfies SI and $\mathrm{M}$ if and only if

$$
G^{1}(x, w)>G^{1}(z, v) \Longleftrightarrow \frac{w}{x}>\frac{v}{z}
$$

Lemma 1 says that, if we want individual growth values to satisfy scale invariance and monotonicity, then the ranking of growth values has to coincide with the ranking of the ratios of second- to first-period income. The axioms $\mathrm{N}$ and $\mathrm{RP}$ are used to cardinalize this ordering, yielding the following.

Proposition 1 A growth measure $G^{1 R}(x, w)$ satisfies SI, M, N, and RP if and only if there exists $\beta>0$ such that

$$
G^{1 R}(x, w)=\beta \frac{(w-x)}{x} .
$$

Proposition 1 characterizes a standard measure of individual growth: the proportional difference between the final and the initial income.

2.1.2 A measure of absolute growth Measures of absolute growth satisfy addition invariance: the value of the function $G^{1}$ does not change if the same amount of income is added to both initial and final income. 
AI (Addition Invariance): For all $\theta>0$ and all $x, w \in \mathbb{R}_{0}^{+}:$

$$
G^{1}(x+\theta, w+\theta)=G^{1}(x, w) .
$$

It is easy to obtain the following Lemma.

Lemma 2 For all $x, v, w, z \in \mathbb{R}_{0}^{+}$the individual growth measure satisfies $\mathrm{AI}$ and $\mathrm{M}$ if and only if

$$
G^{1}(x, w)>G^{1}(z, v) \Longleftrightarrow w-x>v-z .
$$

Lemma 2 says that if we want individual growth values to satisfy addition invariance and monotonicity, then the ranking of growth values has to coincide with the ranking of the differences between second- and first-period income. The axioms N and RP can be used to cardinalize this ordering. This results in the following.

Proposition 2 A growth measure $G^{1 A}(x, w)$ satisfies AI, M, N, and RP if and only if there exists $\alpha>0$ such that

$$
G^{1 A}(x, w)=\alpha(w-x)
$$

Proposition 2 characterizes a standard measure of individual growth: the difference in level between the final and the initial income.

The indices of individual growth obtained in Propositions 1 and 2 have already been introduced in the literature and are widely implemented in empirical works. We provide a unifying framework to derive both indices, using the resourcist principle. Some alternatives that do not satisfy RP are discussed in the footnote following the History-Dependent Growth Incidence axiom in the next section.

\subsection{From individual to aggregate growth}

Let the set $S_{i}=\left\{j \in N\right.$ such that $\left.x_{j}=x(i)\right\}$ contain all individuals that have the $i$-th highest level of income $x(i)$, and let $n_{i}$ be the cardinality of $S_{i}$. The number of different first-period incomes is denoted by $m$.

A first axiom that we impose is a monotonicity axiom, applied to the domain $\hat{D}^{n}$. When comparing two growth processes, the growth process $G^{n}(\mathbf{x}, \mathbf{w})$ has no lower growth than $G^{n}(\mathbf{v}, \mathbf{z})$ if all individuals that occupy the same position in $\mathbf{x}$ and $\mathbf{v}$ experience higher or equal growth in $G^{n}(\mathbf{x}, \mathbf{w})$ than in $G^{n}(\mathbf{v}, \mathbf{z})$. Moreover, if at least one individual experiences higher growth in $G^{n}(\mathbf{x}, \mathbf{w})$ than in $G^{n}(\mathbf{v}, \mathbf{z})$, then aggregate growth has strictly increased.

Rank-Dependent Monotonicity (RDM): $\quad$ For all $n \in \mathbb{N}_{0,1}$, every $G^{n}: \hat{D}^{n} \rightarrow \mathbb{R}$, all $(\mathbf{x}, \mathbf{w})$ and $(\mathbf{v}, \mathbf{z}) \in \hat{D}^{n}$ such that given some $j \in N, G^{1}\left(x_{i}, w_{i}\right)=G^{1}\left(v_{i}, z_{i}\right)$ for all $i \neq j$, and $G^{n}(\mathbf{x}$, $\mathbf{w}) \geq G^{n}(\mathbf{v}, \mathbf{z})$ if and only if $G^{1}\left(x_{j}, w_{j}\right) \geq G^{1}\left(v_{j}, z_{j}\right)$.

This axiom requires that, over the domain $\hat{D}^{n}$, aggregate growth is a strictly increasing function of every individual's growth value. It also implies that apart from the individuals' growth values, the only thing that matters is the rank order and not the income level in the initial income distribution.

We can now formally define the history-dependent growth incidence axiom.

History Dependent Growth Incidence (HDGI): $\quad$ For all $n \in \mathbb{N}_{0,1}$, every $G^{n}: \hat{D}^{n} \rightarrow \mathbb{R}$, and all $(\mathbf{x}, \mathbf{w})$ and $(\mathbf{x}, \mathbf{z}) \in \hat{D}^{n}$ such that given some $k$ and $l \in N, G^{1}\left(x_{i}, w_{i}\right)=G^{1}\left(x_{i}, z_{i}\right)$ for all 
$i \neq k, l$ and there exists a $\Delta>0$ such that $G^{1}\left(x_{l}, z_{l}\right)=G^{1}\left(x_{l}, w_{l}\right)+\Delta$ and $G^{1}\left(x_{k}, z_{k}\right)=$ $G^{1}\left(x_{k}, w_{k}\right)-\Delta$, we have that

$$
\begin{aligned}
& \text { (a) if } x_{l} \leq x_{k} \text {, then } G^{n}(\mathbf{x}, \mathbf{w}) \leq G^{n}(\mathbf{x}, \mathbf{z}), \\
& \text { (b) if } x_{l}=x_{k} \text {, then } G^{n}(\mathbf{x}, \mathbf{w})=G^{n}(\mathbf{x}, \mathbf{z}) .
\end{aligned}
$$

Part (a) requires that aggregate growth does not decrease if individual growth is redistributed from an initially richer to an initially poorer individual. Part (b) requires that aggregate growth is not sensitive to redistributions of individual growth between individuals that have the same initial income level. It is a natural axiom as, from the history-dependent perspective, there is no reason to treat the growth values of those having the same initial income level differently. This ensures that we are only concerned with history dependency, and not with the inequality between individuals' growth values per se, which is the concern in Demuynck and Van de gaer (2012). Moreover, in view of RDM, part (b) implies that if there are several individuals with the same initial income level, only the sum of the their individual growth values matters. Hence, we reformulate the domain by replacing the individual growth values $g_{i}$ by $\bar{g}_{i}=\frac{1}{n_{i}} \sum_{j \in S_{i}} G^{1}\left(x_{j}, w_{j}\right)$, the average of individual growth values of those having the same initial income as individual $i$. Combined with individual growth values that satisfy RP (and that are linear in second period income), this implies zero aversion with respect to second-period income inequalities between individuals that have the same initial income level. ${ }^{2}$

Formally, for each $n \in \mathbb{N}_{0}$ we work with the domain

$$
D^{n}=\left\{\left(\bar{g}_{1}, \ldots, \bar{g}_{n}\right) \in \mathbb{R}^{n} \text { such that } x_{1} \geq \ldots \geq x_{n} \text { and } \bar{g}_{i}=\frac{1}{n_{i}} \sum_{j \in S_{i}} G^{1}\left(x_{j}, w_{j}\right)\right\} .
$$

Every income vector $(\mathbf{x}, \mathbf{z}) \in \hat{D}^{n}$ has a unique representation in $D^{n}$, and all relevant information necessary for the history-dependent perspective is present in the definition of this domain. We are interested in continuous and increasing real valued functions $G^{n}$ over the domain $\hat{D}^{n}$, and continuous and increasing real valued functions $W^{n}$ over the domain $D^{n}$ that are defined as $W^{n}(\overline{\mathbf{g}}(\mathbf{x}, \mathbf{z}))=G^{n}(\mathbf{x}, \mathbf{z})$.

Hence, thanks to the RDM and HDGI axioms, we can work with the domain $D^{n}$. The structural axioms we use (RDM and the three axioms that follow) have been used in the literature, but on different domains. Bossert (1990) used these axioms on the domain of ordered single period income vectors (individual incomes ordered from high to low) to characterize the single series Gini social evaluation function. Demuynck and Van de gaer (2012) used them on the domain of ordered mobility vectors (individual mobilities ordered from high to low). We translate these structural axioms to the domain $D^{n}$.

The Relative Invariance axiom says that comparisons between aggregate growth measures remain invariant when all elements of $\bar{g}$ are multiplied by the same constant.

Relative Invariance (RI): For all $n \in \mathbb{N}_{0,1}$, every $W^{n}: \mathcal{D}^{n} \rightarrow \mathbb{R}$, all $\overline{\mathbf{g}}$ and $\overline{\mathbf{g}}^{\prime} \in \mathcal{D}^{n}$ and $\lambda \in \mathbb{R}_{0}^{+}$, if $W^{n}(\overline{\mathbf{g}})=W^{n}\left(\overline{\mathbf{g}}^{\prime}\right)$, then $W^{n}(\lambda \overline{\mathbf{g}})=W^{n}\left(\lambda \overline{\mathbf{g}}^{\prime}\right)$.

2 In the case one wants to incorporate inequality aversion with respect to the second period income distribution among those that have the same initial income level, one can drop RP and take a measure of individual income growth that is a concave function of second period incomes, such as $G^{1 F O}(x, w)=\log (w)-\log (x)$, proposed by Fields and Ok (1999b), or $G^{1 S V}(x, w)=(w / x)^{r}$ with $0<r<1$, proposed by Schluter and Van de gaer (2011). 
As a result of RDM and RI, the aggregate growth index will be homothetic in $\overline{\mathbf{g}}$ on $D^{n}$.

The next axiom says that comparisons between aggregate growth measures remain invariant when the same constant is added to all elements of $\overline{\mathbf{g}}$.

Translation Invariance (TI): $\quad$ For all $n \in \mathbb{N}_{0,1}$, every $W^{n}: \mathcal{D}^{n} \rightarrow \mathbb{R}$, all $\overline{\mathbf{g}}$ and $\overline{\mathbf{g}}^{\prime} \in D^{n}$ and $\lambda \in \mathbb{R}$, if $W^{n}(\overline{\mathbf{g}})=W^{n}\left(\overline{\mathbf{g}}^{\prime}\right)$, then $W^{n}(\overline{\mathbf{g}}+\lambda \cdot \mathbf{1})=W^{n}\left(\overline{\mathbf{g}}^{\prime}+\lambda \cdot \mathbf{1}\right)$.

As a result of RDM and TI, the aggregate growth index will be translatable in $\overline{\mathrm{g}}$ on $D^{n}$, meaning that all the iso-aggregate growth curves have the same shape, shifted by a constant $\lambda$ in each direction.

The following axiom says that aggregate growth depends on the aggregate growth measure of the group of $n-1$ individuals that have the highest initial incomes, and the growth value of the individual that has the lowest initial income. We can formulate the axiom as follows.

Highest Initial Income Groups Separability (HIIGS): The sequence of aggregate growth measures $\left\{W^{n}\right\}_{n \in \mathbb{N}_{0,1}}$, is such that for every $W^{n}: \mathcal{D}^{n} \rightarrow \mathbb{R}$, all $\overline{\mathbf{g}}$ and $\overline{\mathbf{g}}^{\prime} \in D^{n}$, if $W^{n-1}\left(\bar{g}_{1}, \ldots\right.$, $\left.\bar{g}_{n-1}\right)=W^{n-1}\left(\bar{g}_{1}^{\prime}, \ldots, \bar{g}_{n-1}^{\prime}\right)$ and $\bar{g}_{n}=\bar{g}_{n}^{\prime}$, then $W^{n}(\overline{\mathbf{g}})=W^{n}\left(\overline{\mathbf{g}}^{\prime}\right)$.

The axiom requires that, within all highest initial income groups the trade-offs between the growth values of individuals with different initial incomes remains the same as more and more growth values of individuals with lower initial incomes are added. A similar axiom has been used by Bossert (1990) and Demuynck and Van de gaer (2012).

The combination of the previous axioms results in the following Lemma.

Lemma 3 The sequence of aggregate growth measures $\left\{G^{n}\right\}_{n \in \mathbb{N}_{0.1}}, G^{n}: \hat{D}^{n} \rightarrow \mathbb{R}$ satisfies RDM, HDGI, RI, TI, and HIIGS if and only if there exist numbers $a_{1}, a_{2}, \ldots$ with $a_{1}=1$, and $a_{i} \leq a_{i+1}$ for all $i \in \mathbb{N}_{0}$, such that, for all $(\mathbf{x}, \mathbf{w}) \in \hat{D}^{n}$ and corresponding $\mathbf{g} \in \mathbb{R}^{n}$ with, for all $i \in N, \bar{g}_{i}=\frac{1}{n_{i}} \sum_{j \in S_{i}} G^{1}\left(x_{j}, w_{j}\right)$,

$$
G^{n}(\mathbf{x}, \mathbf{w})=\frac{\sum_{i=1}^{n} a_{i} \bar{g}_{i}}{\sum_{i=1}^{n} a_{i}} .
$$

The Lemma says that aggregate growth can be written as a single series Gini weighted average of individuals' growth values. The weights depend on the individuals' rank in the domain $D^{n}$.

Our final axiom is a standard Population Invariance axiom; it states that the measure of aggregate growth is invariant to a $k$-fold replication of the same vector of initial and final incomes. This property ensures that we can apply this measure to compare growth processes taking place over distributions with different population sizes.

Population Invariance (PI): The sequence of aggregate growth measures $\left\{W^{n}\right\}_{n \in \mathbb{N}_{0}}, W^{n}$ : $\mathcal{D}^{n} \rightarrow \mathbb{R}$ is such that, for all $n, k \in \mathbb{N}_{0}$,

$$
\begin{aligned}
\text { if } \overline{\mathbf{g}} \in D^{n} \text { and } \overline{\mathbf{g}}^{\mathbf{k}} & =(\underbrace{\bar{g}_{1}, \bar{g}_{1}, \ldots, \bar{g}_{1}}_{k \text { times }}, \ldots, \underbrace{\bar{g}_{n}, \bar{g}_{n}, \ldots, \bar{g}_{n}}_{k \text { times }}) \in D^{k n}, \\
\text { then } W^{n}(\overline{\mathbf{g}}) & =W^{k n}\left(\overline{\mathbf{g}}^{\mathbf{k}}\right) .
\end{aligned}
$$


Following Donaldson and Weymark (1980), population invariance allows us to get a functional form for the single series weights. The formal proposition is as follows.

Proposition 3 The sequence of aggregate growth measures $\left\{G^{n}\right\}_{n \in \mathbb{N}_{0}}, G^{n}: \hat{D}^{n} \rightarrow \mathbb{R}$ satisfies RDM, HDGI, RI, TI, HIIGS, and PI if and only if there exists a parameter $\delta \geq 1$, such that, for all $(\mathbf{x}, \mathbf{w}) \in D^{n}$ and corresponding $\overline{\mathbf{g}} \in \mathbb{R}^{n}$ with, for all $i \in N, \bar{g}_{i}=\frac{1}{n_{i}} \sum_{j \in S_{i}} G^{1}\left(x_{j}, w_{j}\right)$,

$$
G^{n}(\mathbf{x}, \mathbf{w})=\sum_{i=1}^{n} \frac{i^{\delta}-(i-1)^{\delta}}{n^{\delta}} \bar{g}_{i} .
$$

Our index of history-dependent growth attaches to each $\bar{g}_{i}$ a weight that is decreasing in the rank of the individual in the initial income distribution. The weights are those derived for the single-series Gini by Donaldson and Weymark (1980). The parameter $\delta$ is a sensitivity parameter: for $\delta=1$, everybody's growth value gets the same weight; as $\delta$ increases, the relative weight of the initially poorest increases and the weight to the initially richest decreases; $\delta=2$ gives the standard Gini weights and as $\delta$ approaches $\infty$, only the growth value of the initially poorest matters.

The value of the index derived in Proposition 3 depends on the value of the sensitivity parameter $\delta$. Abusing notation, we write the index as $G^{n}(\delta)$ to make this dependency explicit. The index is sensitive to both the distribution of growth values among the individuals and the mean of individuals' growth values: doubling all individual growth values does not affect the distribution of growth, but doubles the value of $G^{n}(\delta)$. As advocated by Jenkins and Van Kerm (2011), it is interesting to separate the purely distributive effect of the growth process (the 'progressivity aspect') from the mean growth experienced by the population as a whole. Since $G^{n}(1)$ equals the mean of all individuals' growth values, a natural measure of this progressivity is

$$
P^{n}(\delta)=G^{n}(\delta)-G^{n}(1) .
$$

The progressivity index allows us to compare growth processes' purely distributive effects.

It is instructive to determine the total weights attached to the mean growth rate associated with each initial income level. To this end, suppose that the individuals ranked between $l$ and $k$ share the same initial income level such that for $l \leq i \leq k$, we have that $\bar{g}_{i}=\tilde{g}$. The total weight attached to $\tilde{g}$ in the sum defined in Proposition 3 equals

$$
\frac{1}{n^{\delta}} \sum_{i=l}^{k}\left(i^{\delta}-(i-1)^{\delta}\right)=\frac{1}{n^{\delta}}\left(k^{\delta}-(l-1)^{\delta}\right) .
$$

Hence, after defining $g(i)=\frac{1}{n_{i}} \sum_{j \in S_{i}} g_{j}$, the mean growth rate of all sharing the $i$ - the highest level of initial income, the index can be rewritten as follows:

$$
\sum_{i=1}^{m} \frac{\left[\sum_{j=1}^{i} n_{j}\right]^{\delta}-\left[\sum_{j=1, i \geq 2}^{i-1} n_{j}\right]^{\delta}}{n^{\delta}} g(i) .
$$

Consider the following first-order approximation:

$$
\left[\sum_{j=1, i \geq 2}^{i-1} \frac{n_{j}}{n}\right]^{\delta} \approx\left[\sum_{j=1}^{i} \frac{n_{j}}{n}\right]^{\delta}-\delta\left[\sum_{j=1}^{i} \frac{n_{j}}{n}\right]^{\delta-1} \frac{n_{i}}{n} .
$$


Using this approximation in the expression preceding it, we find the following approximate value for the index:

$$
\sum_{i=1}^{m} \delta\left[\sum_{j=1}^{i} \frac{n_{j}}{n}\right]^{\delta-1} \frac{n_{i}}{n} g(i),
$$

which is the discrete version of the Jenkins and Van Kerm (2011) index. Hence, the Jenkins and Van Kerm index is an approximation of our index. The advantage of our measure, however, is that it works with discrete data, which is the format of all empirical data; it does not require an arbitrary division of the initial income distribution in quantiles and/or its computation does not require the non-trivial estimation of the na-GIC.

Finally, Genicot and Ray (2013) propose the following index:

$$
G R(\epsilon)=\sum_{i=1}^{n} \frac{x_{i}^{-\epsilon}}{\sum_{j=1}^{n} x_{j}^{-\epsilon}} g_{i}
$$

with $\epsilon>0$, a parameter determining the weight that the measure gives to the initially poor. Observe that, for $\epsilon=0$, we obtain the unweighted average of individual growth values (like for our index, when $\delta=1$ ), while for $\epsilon$ approaching infinity, only the growth rate of the initially poorest matters (like for our index when $\delta$ approaches infinity). In terms of axiomatic properties, this index satisfies HDGI, RI, HIIGS, and PI, but not TI nor RDM. Hence, it is also a measure of history-dependent growth, but one that satisfies a more classical (and stronger) separability assumption. The disadvantage of the Genicot and Ray index is that it is less directly related to the na-GIC, which only contains information on growth rates and their ranking in the initial income distribution, not on their initial income level.

\section{The distributional implications of the crisis in Italy}

In this section we implement our theoretical framework in order to investigate changes in the Italian growth process over the last decade. Using our indices $G^{n}(\delta)$ and $P^{n}(\delta)$, we assess the consequences of the recent economic crisis on the Italian growth process from the history-dependent perspective.

\subsection{The data}

Our empirical illustration is based on the panel component of the last seven waves of the Bank of Italy's Survey on Household Income and Wealth (SHIW). The SHIW is a representative sample of the Italian resident population interviewed every two years. In particular, we consider the 1998, 2000, 2002, 2004, 2006, 2008, and 2010 waves.

The unit of observation is the household, defined as all persons sharing the same dwelling. Our measure of living standard is household net disposable income, which includes all household earnings, transfers, pensions, and capital incomes, net of taxes and social security contributions. Household income is expressed in constant prices of 2010, and to adjust for differences in household size, divided by the square root of household size. In line with the literature, for each wave we drop the bottom and top $1 \%$ in the 
Table 1. History-dependent growth indices 2004-06 and 2008-10

\begin{tabular}{llllll}
\hline & \multicolumn{6}{l}{$\delta$} & & \\
\cline { 2 - 6 } & 1 & 2 & 4 & 6 & 8 \\
\hline Panel A: relative growth & & & & & \\
$G_{04 / 06}^{n}(\delta)$ & 0.0641 & 0.1128 & 0.1673 & 0.2053 & 0.2366 \\
$G_{08 / 10}^{n}(\delta)$ & 0.0240 & 0.0523 & 0.0810 & 0.0994 & 0.1136 \\
$G_{04 / 06}^{n}(\delta)-G_{08 / 10}^{n}(\delta)$ & $0.0401^{* * *}$ & $0.0606^{* * *}$ & $0.0863^{* * *}$ & $0.1059^{* * *}$ & $0.1230^{* * *}$ \\
Panel B: absolute growth & & & & & \\
$G_{04 / 06}^{n}(\delta)$ & 425.2 & 1038.5 & 1335.9 & 1485.8 & 1597.6 \\
$G_{08 / 10}^{n}(\delta)$ & -41.90 & 432.2 & 635.8 & 716.0 & 767.6 \\
$G_{04 / 06}^{n}(\delta)-G_{08 / 10}^{n}(\delta)$ & $467.1^{* * *}$ & $606.3^{* * *}$ & $700.1^{* * *}$ & $769.8^{* * *}$ & $830.0^{* * *}$ \\
\hline
\end{tabular}

Notes: In the third line of both panels, asterisks ***, **, and * indicate the $1 \%, 5 \%$, and $10 \%$ levels of significance, respectively.

income distribution from the sample to eliminate the effect of possible outliers. This results in between 3,393 (for 2002-04) and 4,395 (for 2008-10) observations. ${ }^{3}$

To investigate the distributional impact of the recent economic crisis, we use the growth process 2008-10 as benchmark since the first wave of the crisis took place in Italy in 2008. We compare all previous two-year period growth processes starting from 1998-00 with this benchmark. For the sake of brevity, we only provide a detailed report for the comparison with the 2004-06 period, which immediately preceded the economic crisis. Apart from the crisis, these adjacent periods are most similar. These periods differ in terms of the growth in mean income and the mean of individuals' income growth, but our progressivity index eliminates the latter effect. The comparisons of the other periods with the benchmark yield broadly similar results and are briefly discussed at the end of the next section; the detailed results are reported in Appendix C.2 for completeness.

We use sample weights to compute all estimates. ${ }^{4}$ We give each household the sample weight corresponding to the sampling in the first wave of the survey in our analysis (1998). To the households selected into the survey at subsequent waves, we give the sample weight corresponding to the sampling in the wave of their first inclusion into the survey. ${ }^{5}$ The standard errors of our estimates are obtained through 1,000 bootstrap replications-see Appendix B for more details.

\subsection{Results}

In this section we first compare the growth process 2004-06 against the growth process 2008-10. Remember from panel (b) in Fig. 2 that the cumulative individual na-GICs cross. Table 1 reports and compares the numerical values of our index for both growth processes.

3 For more information about sample sizes, see Table C.1 in Appendix C.1.

4 We use cross-sectional individual sample weights at time $t$. As shown by Faiella and Gambacorta (2007) in the case of the SHIW, for the production of longitudinal statistics, there is no unambiguous evidence that the use of longitudinal weights always performs better than cross-sectional weighing in terms of efficiency.

5 See Hildebrand et al. (2012) and Jenkins and Van Kerm (2011). 
Table 2. History-dependent progressivity indices 2004-06 and 2008-10

\begin{tabular}{lllll}
\hline & \multicolumn{5}{l}{$\delta$} & \\
\cline { 2 - 5 } & 2 & 4 & 6 & 8 \\
\hline $\begin{array}{l}\text { Panel A: relative index } \\
P_{04 / 06}^{n}(\delta)\end{array}$ & 0.0487 & 0.1032 & 0.1412 & 0.1725 \\
$P_{08 / 10}^{n}(\delta)$ & 0.0283 & 0.0570 & 0.0754 & 0.0896 \\
$P_{04 / 06}^{n}(\delta)-P_{08 / 10}^{n}(\delta)$ & $0.0204^{*}$ & $0.0462^{* *}$ & $0.0658^{* *}$ & $0.0829^{* * *}$ \\
$P_{\text {Panel B: absolute index }}$ & & & & \\
$P_{04 / 06}^{n}(\delta)$ & 613.3 & 910.7 & 1060.6 & 1172.4 \\
$P_{08 / 10}^{n}(\delta)$ & 474.1 & 677.7 & 757.9 & 809.5 \\
$P_{04 / 06}^{n}(\delta)-P_{08 / 10}^{n}(\delta)$ & 139.2 & $233^{*}$ & $302.7^{* *}$ & $362.9^{* *}$ \\
\hline
\end{tabular}

Notes: In the third line of both panels, asterisks ***, **, and * indicate the $1 \%, 5 \%$, and $10 \%$ levels of significance, respectively.

For all values of the sensitivity parameter $\delta$ given in the table, our index measures more history-dependent growth during the 2004-06 process than during the 2008-10 process. This holds both when a relative and an absolute index is used. The difference is always statistically significant. Hence, it can be inferred that the 2004-06 growth episode is better than the 2008-10 episode according to our measure of history-dependent (relative and absolute) growth. Focusing on the value of the index when $\delta=1$, that is when all individuals' growth values get the same weight, the difference between the indices of the two processes is already substantial. This might imply that the result when $\delta>1$ is mostly due to the different overall levels of growth. Hence, we adopt the solution given at the end of Section 2.3 and compute the progressivity indices $P^{n}(\delta)$ to compare the pure distributional effect of both processes.

The results, reported in Table 2, show that also when the focus is on the pure distributional effect of growth, the 2004-06 growth process is preferred from a history-dependent perspective to the 2008-10 growth process (although the result is not significant for the absolute index when $\delta=2$, is only significant at $10 \%$ for the relative index when $\delta=2$, and for the absolute index when $\delta=4$ ). Thus, both the overall extent of growth and the pure distributional effect play a role in the history-dependent ranking of the growth process 2004-06 above the growth process 2008-10.

In order to put the distributional implications of the crisis into further perspective, it is interesting to compare the crisis to the other previous growth processes (1998-00, 2000-02, 2002-04)—see Table C.2 in Appendix C.2. In fact, all these periods performed better than the 2008-10 period from a history-dependent growth perspective, irrespective of whether the relative or the absolute growth index is used. Even when only the distributional aspect is considered, this conclusion holds true. This is illustrated in Table 3, which gives the proportional change in mean income, the mean of individuals' proportional income change, $G(1)$, and the values of the relative progressivity measure for each of the periods. ${ }^{6}$

Comparing the rankings of the growth processes on the basis of the second and third columns in Table 3, we see only one re-ranking: the 2004-06 growth process ranks secondbest on the basis of proportional change in mean income, and third-best on the basis of $G(1)$. The opposite occurs for the 2002-04 growth process. This indicates that the 2004-06

6 The results are very similar when the absolute progressivity index is used. 
Table 3. Relative progressivity index $P^{n}(\delta)$ for each process

\begin{tabular}{lllllll}
\hline & \multirow{2}{*}{$\begin{array}{l}\text { Proportional change } \\
\text { in mean income }\end{array}$} & $G(1)$ & \multicolumn{2}{l}{$P(\delta)$} & & \\
\cline { 5 - 7 } & & & $\delta=2$ & $\delta=4$ & $\delta=6$ & $\delta=8$ \\
\hline $98 / 00$ & 0.0230 & 0.0963 & 0.0771 & 0.1858 & 0.2478 & 0.3065 \\
$00 / 02$ & 0.0159 & 0.0612 & 0.0492 & 0.1048 & 0.1449 & 0.1788 \\
$02 / 04$ & 0.0181 & 0.0692 & 0.0576 & 0.1305 & 0.1841 & 0.2279 \\
$04 / 06$ & 0.0207 & 0.0641 & 0.0487 & 0.1032 & 0.1412 & 0.1725 \\
$08 / 10$ & -0.0021 & 0.0240 & 0.0283 & 0.0570 & 0.0754 & 0.0896 \\
\hline
\end{tabular}

process is less in favor of the initially poor than the 2002-04 process, because the proportional change in mean income can be written as a weighted average of individuals' proportional income change, with individuals' weight equal to their share in the initial distribution of income, while the mean of individuals' proportional income change weights all individuals' proportional income change equally. It is striking that the ranking of the processes based on the progressivity indices, irrespective of the value for $\delta$, is almost the same as the ranking of the processes based on $G(1)$. The only difference is that the 2004-06 process drops one position in the ranking and becomes worse than the 2000-02 process. This confirms that, relative to the other periods before the crisis, the 2004-06 process is characterized by a poor distribution of growth from a history-dependent perspective. Hence, even when compared to the period with the worst distribution of growth before the crisis (2004-06), the period after the crisis performed bad from a history-dependent perspective.

We conclude our analysis by performing some robustness checks related to variation in household composition. First, it might be argued that the results of our analysis are sensitive to changes in household composition between the initial (first) period and the second period of the growth process. Hence, we recalculated our estimates using, for each growth process, only the subsample of households that did not change in composition between the initial and second period. The results, reported in Appendix C.3, show that our conclusions are not affected. Second, changes in household composition between different growth processes might also affect our comparisons. Therefore, we adopt three alternative equivalence scales. We look at per capita incomes, which assumes that each household member counts as 1 in the equivalence scale. We also use the old OECD equivalence scale, which assigns a value of 1 to the first household member, 0.7 to each additional adult, and 0.5 to each child. Finally, we use the modified OECD equivalence scale, which assigns a value of 1 to the household head, 0.5 to each additional adult member, and 0.3 to each child. For each of the distributions of growth in household equivalized income corresponding to these equivalence scales, we compute the history-dependent indices in Appendix D. Our main conclusion, that the post-crisis period 2008-10 was the worst from a history-dependent growth perspective, even when only the distribution of growth is considered, is not sensitive to the choice of the equivalence scale.

\subsection{Comparison with other growth indices}

It is interesting to compare $G(\delta)$ with some of the indices that have been provided by the literature and that are related to our framework. We consider the history-dependent indices 
introduced by Genicot and Ray (2013) (see eq. (1)) and by Jenkins and Van Kerm (2011). The latter is defined for $v \geq 1$ as

$$
J V(v)=\int_{0}^{1} v(1-p)^{v-1} \tilde{g}(p) d p, \text { with } \tilde{g}(p)=\frac{y_{t+1}\left(p_{t}\right)-y_{t}\left(p_{t}\right)}{y_{t}\left(p_{t}\right)},
$$

such that $y_{t+1}\left(p_{t}\right)$ is the income in period $t+1$ of those that were at percentile $p$ at time $t$; and $\tilde{g}(p)$ is an estimate of the na-GIC.

In addition, we consider the indices introduced by Ravallion and Chen (2003) and Essama-Nssah (2005), which are the main indices used to measure growth in the standard anonymous setting. The Essama-Nssah index is defined for $v \geq 1$ as:

$$
\operatorname{EN}(v)=\int_{0}^{1} v(1-p)^{v-1} g(p) d p, \text { with } g(p)=\frac{y_{t+1}\left(p_{t+1}\right)-y_{t}\left(p_{t}\right)}{y_{t}\left(p_{t}\right)},
$$

such that $y_{t+1}\left(p_{t+1}\right)$ is the income in period $t+1$ of those that are at percentile $p$ at time $t+1 ; g(p)$ is an estimate of the GIC. This index can be considered as the anonymous counterpart of the Jenkins and Van Kerm index. In fact, it is a weighted average of the coordinates of the GIC, with weights that depend on the 'anonymous percentiles'.

The Ravallion and Chen (2003) index is defined as

$$
R C=\frac{1}{H_{t}} \int_{0}^{H_{t}} g(p) d p,
$$

where $H_{t}$ is the poverty headcount ratio of the initial distribution. Index $R C$ focuses on the group of individuals below the poverty line and measures the average growth rate of these individuals.

Table 4 lists the different rankings of the growth processes for the different indices (the detailed results are reported in Appendix F).

For all relative indices, the $1998-00$ period is ranked best, while the $2008-10$ period is ranked worst. For all absolute indices, the worst period is 2008-10. Hence, a first important and robust observation is that, for all indices, the crisis period 2008-10 was the worst in recent Italian history. Moreover, the difference with the crisis period is highly statistically significant for all growth indices, irrespective of whether an absolute or a relative index was used (see Appendix E). Also when abstracting from the differences in mean growth rates, which can be done for our index and the Jenkins and Van Kerm index, the crisis years were the worst. With a relative progressivity index, the difference with the other periods is almost always highly statistically significant, and with an absolute progressivity index the difference is often statistically significant.

The observation that all the indices agree that the 2008-10 is the worst growth episode does not imply that it never makes any difference which index is used. When one considers the periods before the crisis, the different indices often lead to different rankings of the growth processes.

When the focus is on relative growth, the ranking in the second and fourth row of Panel A occurs for the history-dependent growth indices, but not for the anonymous indices. Hence, taking a history-dependent or an anonymous perspective matters. Moreover, within the set of history-dependent growth indices, the rank-dependent measures $\left(G^{n}(\delta)\right.$ and $J V(v))$ behave differently from the $G R(\epsilon)$ measure, where the same ranking occurs for low $(\epsilon \leq 0.2)$ and higher $(3.8 \leq \epsilon \leq 5.2)$ values of $\epsilon$, while another ranking occurs for intermediate values. Moreover, the rankings for high values of $\epsilon(\epsilon \geq 5.4)$ are not encountered 
Table 4. Ranking of the growth processes

\begin{tabular}{|c|c|c|c|c|c|c|c|}
\hline \multirow{3}{*}{ Ranking } & \multicolumn{7}{|l|}{ Index } \\
\hline & \multicolumn{4}{|c|}{ History dependent } & \multirow[b]{2}{*}{$G R(\epsilon)$} & \multicolumn{2}{|l|}{ Anonymous } \\
\hline & $G^{n}(\delta)$ & $P^{n}(\delta)$ & $J V(v)$ & $P_{J V}(v)$ & & $E N(v)$ & $R C$ \\
\hline \multicolumn{8}{|l|}{ Panel A: relative index } \\
\hline $04 / 06,02 / 04,00 / 02$ & $\times$ & $\times$ & $\times$ & $\times$ & $5.4 \leq \epsilon \leq 7.6$ & $v=1$ & $\times$ \\
\hline $04 / 06,00 / 02,02 / 04$ & $\times$ & $\times$ & $\times$ & $\times$ & $\epsilon \geq 7.8$ & $\times$ & $\times$ \\
\hline $02 / 04,04 / 06,00 / 02$ & $\delta \leq 5.2$ & $\delta \leq 1.4$ & $v \leq 1.6$ & $x$ & $\epsilon \leq 0.2,3.8 \leq \epsilon \leq 5.2$ & $v \geq 1.2$ & $\vee$ \\
\hline $02 / 04,00 / 02,04 / 06$ & $\delta \geq 5.4$ & $\delta \geq 1.6$ & $v \geq 1.8$ & $v \geq 1.2$ & $0.4 \leq \epsilon \leq 3.6$ & $\times$ & $\times$ \\
\hline \multicolumn{8}{|l|}{ Panel B: absolute index } \\
\hline $04 / 06,98 / 00,02 / 04,00 / 02$ & $\times$ & $\times$ & $\times$ & $\times$ & $5.2 \leq \epsilon \leq 7.8$ & $v \leq 1.2$ & $\times$ \\
\hline $98 / 00,04 / 06,02 / 04,00 / 02$ & $\delta \leq 2.4$ & $\delta \leq 1.2$ & $v \leq 3$ & $v \leq 1.2$ & $\epsilon \leq 0.6,3.6 \leq \epsilon \leq 5.0$ & $1.4 \leq v \leq 4.4$ & $\times$ \\
\hline $98 / 00,02 / 04,04 / 06,00 / 02$ & $\delta \geq 2.6$ & $\delta \geq 1.4$ & $v \geq 3.2$ & $v \geq 1.4$ & $0.8 \leq \epsilon \leq 3.4$ & $4.6 \leq v \leq 6.8$ & $\vee$ \\
\hline $02 / 04,98 / 00,04 / 06,00 / 02$ & $\times$ & $\times$ & $\times$ & $\times$ & $\times$ & $v \geq 7$ & $\times$ \\
\hline $04 / 06,98 / 00,00 / 02,02 / 04$ & $\times$ & $x$ & $\times$ & $\times$ & $\epsilon \geq 8.0$ & $\times$ & $\times$ \\
\hline
\end{tabular}

Notes: In Panel A, the best growth process is always 1998-00, while the worst is 2008-10. Hence, the panel only lists the three other growth processes, in decreasing order. In Panel B, the worst period is 2008-10. Hence, the panel only lists the four other growth processes, in decreasing order. Entry ' $x$ ' means that the ranking does not occur for the index and entry, while ' $V$ ' means that the ranking is the only ranking that occurs for the index. Rankings are determined by grid search, in which values of the parameters are increased in steps of 0.2 , from 1 to 8.8 for $\delta$ and $v$, and from 0 to 8.8 for $\epsilon$. Further, $J V(v)$ is obtained by estimating the covariancebased expressions for the generalized concentration coefficients, applied on the distribution of individual growth ordered on the basis of the rank of individuals in the initial distribution, which involves estimating the initial fractional ranks and the sample covariance between the individual growth measures (divided by their sample mean) and those (fractional) ranks (see Van Kerm 2010). Moreover, GR $(\epsilon)$ is obtained by applying the index formulation (1) on the data as they appear in the dataset; $E N(v)$ is obtained by fist estimating the coordinates of the GIC and then aggregating them using the rank-dependent procedure in Essama-Nssah (2005). Relative $R C$ is obtained as minus the variation of the Watts index of poverty divided by the initial headcount ratio (see Ravallion and Chen 2003). Absolute $R C$ is obtained as minus the variation of the average poverty gap not normalized by the poverty line, divided by the initial headcount ratio. For both relative and absolute $R C$ we use the official relative poverty line in 2008 expressed in constant 2010 prices.

for the other history-dependent indices. Finally, observe that the ranking in the third row changes into the one in the fourth row for much lower values of the parameter for the $J V(v)$ than for the $G^{n}(\delta)$ index. If, for instance, the weights of the standard Gini index are taken (i.e. $\delta=v=2$ ), the two indices disagree on the ranking of 2004-06 versus 2000-02.

When the focus is on absolute growth, the ranking in the fourth row of Panel B in the table only occurs for $E N(v)$ and never for the history-dependent indices. Hence, again, taking a history-dependent or an anonymous perspective matters. Within the set of historydependent growth indices, the same patterns occur as for relative growth indices. The rankdependent measures $\left(G^{n}(\delta)\right.$ and $\left.J V(\nu)\right)$ behave differently from the $G R(\epsilon)$ measure, which gives the same ranking for low $(\epsilon \leq 0.6)$ and for higher $(3.6 \leq \epsilon \leq 5.4)$ values of $\epsilon$, while another ranking occurs for intermediate values. Again, the rankings for high values of $\epsilon(\geq 5.2)$ are not found for the other history-dependent indices. Looking at the $G^{n}(\delta)$ and $J V(v)$ indices, the ranking in the second row changes in the one in the third row for lower values of the parameter for the $J V(v)$ than for the $G^{n}(\delta)$ index, although the difference is less remarkable than for relative growth. 


\section{Conclusions}

The size of the recent economic crisis begs the question of the distributional impact of the crisis. More in particular, we want to know whether the crisis has a greater effect on the initially poor or the initially rich. This is a history-dependent approach since it takes into account individuals' initial economic conditions.

Endorsing this perspective, we provide a characterization of an index of history-dependent growth. The crucial steps in the characterization are the definition of the domain, which allows us to keep track of individuals' position in the initial income distribution and the history-dependent growth axiom, which prefers redistributions of growth to the initially poorest and is indifferent between growth redistributions between individuals having the same initial income. The resulting index of history-dependent growth is expressed as a weighted average of the mean income growth associated with each initial income level, with weights that decrease with the rank of these individuals in the initial distribution of income. Our index turns out to be closely related to the mobility measure of Jenkins and Van Kerm (2011), but is easier to compute, and, like their index, it is additively decomposable into a pure distributive effect and the mean of individuals' income growth. However, our index is characterized using axioms defined for individuals' income, and is computed easily using these individual data. The Jenkins and Van Kerm index (2011) is defined in terms of the naGIC and relies on a non-trivial estimation of this curve. As a result, it is unclear which of the axioms that a history-dependent growth index should have are satisfied by their index. This will depend on the way the na-GIC is estimated.

We have shown the applicability of our framework with an empirical application to the economic crisis in Italy. Individual na-GICs cross frequently, making it impossible to obtain clear conclusions about the ranking of growth processes. Our measure also allows us to obtain a clear ranking in such situations. We find that the growth process during the crisis is worse from a history-dependent perspective than any of the preceding growth processes, even when we correct for the differences in the mean of individuals' income growth and only consider the way growth is distributed. This result is not influenced by changes in household composition between the initial and second period, and is also robust to the choice of the equivalence scale.

Finally, in the empirical application we have compared our index and other measures of both history-dependent and anonymous growth. We have shown that, while they all agree in identifying the years $2008-10$ as the worst growth process, they lead to considerable differences in the ranking of the growth processes prior to the financial crisis.

\section{Supplementary material}

Supplementary material (the Appendix) is available online at the OUP website.

\section{Acknowledgements}

We are grateful to the editor and four anonymous referees for their useful comments. We also thank Paolo Brunori, Thomas Demuynck, François Maniquet, Vito Peragine, Xavi Ramos, and Philippe Van Kerm for their suggestions. We gratefully acknowledge comments received on preliminary versions presented at Universitat Autònoma de Barcelona, Ghent University, CORE, the Fifth ECINEQ meeting (Bari, 22-24/07, 2013), the VI workshop of the GRASS and VI Academia Belgica-Francqui Foundation conference (Rome, 10-12/09, 2012), and the Second World bank 
Conference on Equity: Inequality of What? Outcomes, opportunities and Fairness (Washington, 26-27/06/2012). The paper was largely written when the first author was visiting Universitat Autònoma de Barcelona, and the second author was visiting the Institut d'anàlisi Econòmica (CSIC) in Barcelona. We sincerely thank both institutions for their hospitality.

\section{References}

Atkinson, A.B. and Brandolini, A. (2010) On analyzing the world distribution of income, The World Bank Economic Review, 24, 1-37.

Bossert, W. (1990) An axiomatization of the single-series Gini, Journal of Economic Theory, 50, 82-92.

Bourguignon, F. (2011) Non-anonymous Growth Incidence Curves, Income Mobility and Social Welfare Dominance: a theoretical framework with an application to the global economy, Journal of Economic Inequality, 9, 605-27.

D’Agostino, M. and Dardanoni, V. (2009) What's so special about Euclidean distance? Social Choice and Welfare, 33, 211-33.

Demuynck, T. and Van de gaer, D. (2012) Inequality adjusted income growth, Economica, 79, 747-65.

Donaldson, D. and Weymark, J. (1980) A single-parameter generalization of the Gini indices of inequality, Journal of Economic Theory, 22, 67-86.

Essama-Nssah, B. (2005) A unified framework for pro-poor growth analysis, Economics Letters, $89,216-21$.

Essama-Nssah, B. and Lambert, P. (2009) Measuring pro-poorness: a unifying approach with new results, Review of Income and Wealth, 55, 752-78.

Faiella I. and Gambacorta R. (2007) The weighting process in the SHIW, Banca d'Italia, Temi di discussione del Servizio Studi, Number 636, Rome.

Fields, G.S. and Ok, E.F. (1999a) The measurement of income mobility: an introduction to the literature. In J. Silber (ed), Handbook of Income Inequality Measurement, Kluwer Academic Publishers, Dordrecht, 557-98.

Fields, G.S. and Ok, E.F. (1999b) Measuring movement of incomes, Economica, 66, 455-71.

Genicot, G. and Ray, D. (2013) Measuring upward mobility, paper presented at the fifth ECINEQ meeting in Bari.

Grimm, M. (2007) Removing the anonymity axiom in assessing pro-poor growth, Journal of Economic Inequality, 5, 179-97.

Grosse, M., Harttgen, K., and Klasen, S. (2008) Measuring pro-poor growth in non-income dimensions, World Development, 36, 1021-47.

Hildebrand, V., Alperin, M., and Van Kerm, P. (2012) Measuring and accounting for the deprivation gap of Portuguese immigrants in Luxembourg, CEPS/INSTEAD Working Paper No 2012-33, Luxembourg.

Jenkins, S. and Van Kerm, P. (2006) Trends in income inequality, pro-poor income growth, and income mobility, Oxford Economic Papers, 58, 531-48.

Jenkins, S. and Van Kerm, P. (2011) Trends in individual income growth: measurement methods and British evidence, IZA Discussion Paper. No. 5510, Bonn.

Kakwani, N. and Pernia, E. (2000) What is pro-poor growth? Asian Development Review, 18, $1-16$.

Kakwani, N. and Son, H. (2008) Poverty equivalent growth rate, Review of Income and Wealth, $54,643-55$.

Kolm, S.C. (1976a) Unequal inequalities: I, Journal of Economic Theory, 12, 416-42.

Kolm, S.C. (1976b) Unequal inequalities: II, Journal of Economic Theory, 13, 82-111.

Kraay, A. (2006) When is growth pro-poor? evidence from a panel of countries, Journal of Development Economics, 80, 198-227. 
Palmisano, F. and Peragine, V. (2015) The distributional incidence of growth: a social welfare approach, Review of Income and Wealth, 61, 440-64.

Ravallion, M. and Chen, S. (2003) Measuring pro-poor growth, Economics Letters, 78, 93-99.

Schluter, C. and Van de gaer, D. (2011) Upward structural mobility, exchange mobility, and subgroup consistent mobility measurement, Review of Income and Wealth, 57, 1-22.

Son, H.H. (2004) A note on pro-poor growth, Economics Letters, 82, 307-14.

Van Kerm, P. (2010) sgini Generalized Gini and Concentration coefficients (with factor decomposition) in Stata, CEPS/INSTEAD, available at: http://medim.ceps.lu/stata/sgini.pdf, last accessed on April 2015.

Van Kerm, P. (2009) Income mobility profiles, Economics Letters, 102, 93-5. 